\title{
Ti-6Al-4V microstructural orientation at different length scales as a function of scanning strategies in Electron Beam Melting in additive manufacturing
}

\author{
Priyanka Agrawal ${ }^{1}$, Maria J. Quintana ${ }^{1}$, Matt Kenney ${ }^{1}$, Sabina Kumar $^{2}$, \\ Alec Saville ${ }^{3}$, Amy Clarke ${ }^{3}$, Peter C. Collins ${ }^{1}$ \\ ${ }^{1}$ Iowa State University, Materials Science and Engineering Department, Ames, IA, United States \\ ${ }^{2}$ University of Tennessee, Department of Materials Science and Engineering Department, Knoxville, TN, United States \\ 3 Colorado School of Mines, Metallurgical and Materials Engineering Department, Golden, CO, United States
}

\begin{abstract}
Additive manufacturing has been around for many years, yet the underlying physics of thermal gradients, local pressure environment, and other non-steady state manufacturing conditions are not fully understood. A Multi-University Research Initiative (MURI) is currently ongoing to measure liquid/solid and solid/solid interface stabilities in AM Ti-6Al-4V. Samples were produced with different beamscanning strategies in order to study the role of thermal gradients on the resulting microstructure. The motivation is to determine which beam-scanning strategy leads to desired grain size and texture. Orientation at different length scales (from $\mathrm{mm}$ to $\mathrm{nm}$ ) can be quantified and compared with a combination of techniques including Precession Electron Diffraction (PED), Electron Backscatter Diffraction (EBSD) and Neutron diffraction. This new information will help predict properties of additively manufactured parts.
\end{abstract}

\section{Introduction}

A principal advantage of manufacturing parts by Additive Manufacturing (AM) system is the ability to obtain complex geometries, different types of composites/alloys or even gradients of composition in the same part, as it follows a layer-by-layer build up approach. Therefore, when each layer is been printed, it is possible to melt 4-5 layers below it, as per the thermal conductivities and thermal diffusivities of the material used. This has thusly created the necessity of understanding the relationships between process, composition, microstructure and properties [1-4], as well as creating mathematical and computational models that can predict the properties of the final printed parts [4]. However, in order to do so, it is necessary to fully understand the fundamentals that dictate the microstructure (defects, phase fractions, morphologies, texture, etc.) and mechanical properties as a function of thermal gradients, local pressure environments and solidification strategies (Figure 1) [5-9].

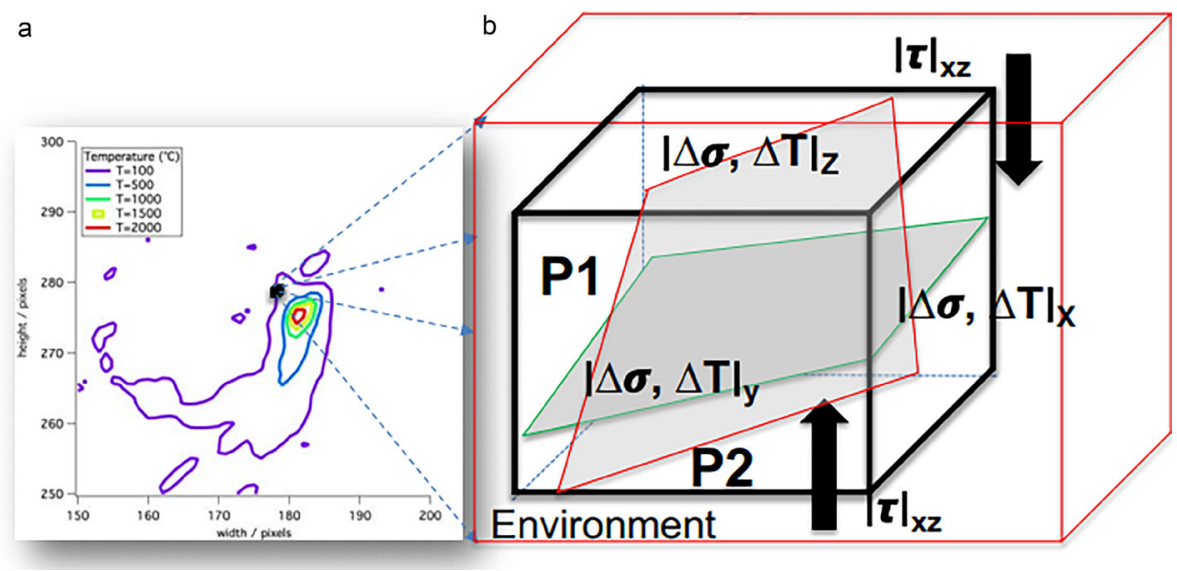

Figure 1: Overview of the scientific problem: (a) Instantaneous temperature contour, during a laser beam scan, on an Alloy 718 build shows asymmetrical distribution; (b) Problem statement: spatial-temporal thermomechanical boundary conditions may trigger complex interface stabilities and defect generations. *Figure created for the MURI ONR project N00014-18-1-2794

The Rosenthal equation [10] gives the three-dimensional steady state temperature field for a point source. While it is a straight forward analytical solution, it does not account for complex boundary conditions and non-steady state conditions, nor for latent heat of fusion or convection. A recent semi-analytical heat model has been developed at Oak Ridge National Laboratory Manufacturing Demonstration Facility (ORNL MDF), to tackle the above-mentioned complexities [11], based on a transient solution, to construct a temperature field for an arbitrary beam path, from which information can be extracted regarding thermal gradients and interphase growth velocities of a layer of a build, both essential in understanding the microstructural features of the build such as phase selection (or variant selection in Ti-6Al$4 \mathrm{~V})$, morphological features of the phase.

Electron-Powder Bed Fusion systems are known to create complex heat and mass transfer conditions that vary throughout the build as the heat source creates a melt pool that moves following a given scanning strategy [12]. When each layer is been printed, it is possible to melt 4-5 layers below it, as per the thermal conductivities and thermal diffusivities of the material used.

(C) The Authors, published by EDP Sciences. This is an open access article distributed under the terms of the Creative Commons Attribution License 4.0 (http://creativecommons.org/licenses/by/4.0/). 
The properties of AM parts, as with any other manufacturing process, are a function of composition, microstructure (phases, grain size and morphology, etc.), orientation, texture, defects (created during the manufacturing process) and superficial characteristics [7]. Each one of these parameters can be analyzed using different equipment and techniques, as shown in Figure 2.

\begin{tabular}{|c|c|c|c|c|}
\hline $\begin{array}{c}\text { Bulk } \\
\text { Composition }\end{array}$ & Optical Imaging & SRAS $*$ & $\begin{array}{c}\text { X-ray } \\
\text { Tomography }\end{array}$ & $\begin{array}{c}\text { Macro } \\
\text { Photography }\end{array}$ \\
$\begin{array}{c}\text { Elemental } \\
\text { Partitioning }\end{array}$ & SEM Imaging * & EBSD $*$ & EBSD/SEM * & $\begin{array}{c}\text { Laser } \\
\text { Interferometry }\end{array}$ \\
Solute Clusters * & TEM Imaging * & $\begin{array}{c}\text { Synchrotron/ } \\
\text { PED }\end{array}$ & PED/TEM * & AFM/STM \\
\hline $\begin{array}{c}\text { Chemical } \\
\text { Composition }\end{array}$ & $\begin{array}{c}\text { Microstructure } \\
\text { Averages }\end{array}$ & $\begin{array}{c}\text { Microstructure } \\
\text { Configuration }\end{array}$ & Defect Analysis & Surface Imaging \\
\hline
\end{tabular}

Figure 2: Diagram of techniques used to characterize different parameters present in AM parts in different scales. Stars represent techniques that are being used by MURI members in this project.

The goal of this Multidisciplinary University Research Initiative (MURI) funded by the Office of Naval Research is to understand how the variation of composition and thermal gradients, both spatially and temporally, will result in differences in liquid-solid interface velocities, thermal gyrations and elastic-plastic stress/strain gradients as a function of geometry and energy deposition while manufacturing the part; in other words understanding how the build parameters influence local conditions that result in specific microstructural features, defects and heterogeneities.

\section{Materials and Experiments}

ORNL MDF additively manufactured Ti-6Al-4V samples with 3 different scan strategies (shown schematically in Figure 3), namely raster (or line scan) (L) and two spot melt scans, random fill (R) and Dehoff fill (D), in order to evaluate and understand the complexities of AM fundamentals previously mentioned, including the spatial and temporal thermo-mechanical distributions in AM parts. The chosen design was to fabricate cuboids that have a wide range of thermal gradients and interface velocities. These cuboids were built on an ARCAM Q10 Plus machine as: $15 \times 15 \times 25 \mathrm{~mm}$ with a layer height of $50 \mu \mathrm{m}$. The builds were made under a vacuum of $4.5 \times 10^{-2} \mathrm{mBar}$ and maintained at a preheat temperature of $470^{\circ} \mathrm{C}$. In this multi-university project, twelve samples of each melting strategy were built concurrently, Iowa State University was provided with samples designated as number 5 (L5, R5 and D5).

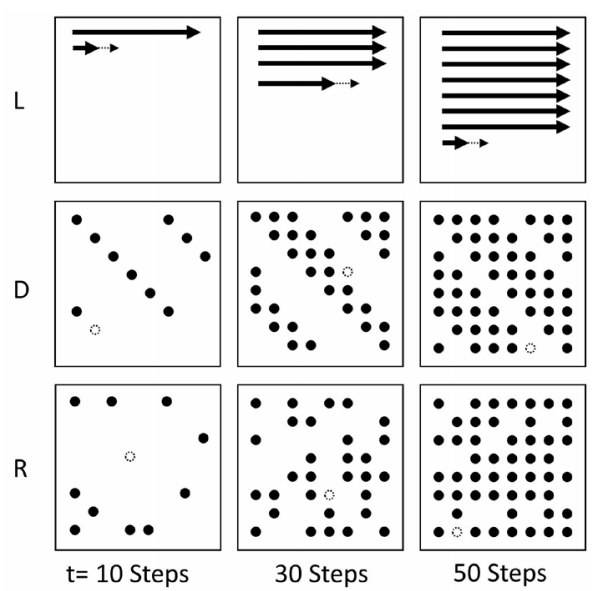

Figure 3: Schematic of the different scanning strategies at different time steps. L is a Raster line scan, D is a Dehoff point melting strategy, and R is a Random point melting strategy. The open circle and smaller line represent the position that will be active in one additional time step.

The composition of the powder from which AM samples were obtained as reported by Timet as nominal composition: Ti, 5.5-6.75\% Al,

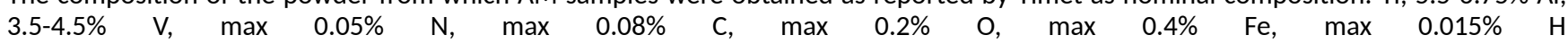
(http://www.timet.com/assets/local/documents/datasheets/alphaandbetaalloys/6-4.pdf).

The samples for microscopy were sectioned using wire cutting - EDM (electrical discharge machining). The cuts were designed to provide access to different cross-sectional planes of the build that would be necessary for imaging using scanning electron microscopy (SEM) and transmission electron microscopy (TEM), electron backscatter diffraction (EBSD), precession electron diffraction (PED), and, in the future, for spatially resolved acoustic spectroscopy (SRAS) analysis. A portion of the samples' XY plane (perpendicular to build direction) and the complete XZ plane (parallel to build direction) were ground and polished using traditional techniques and an overnight polishing on Vibromet (Buehler VibroMet 2 Vibratory Polisher). 
Scanning electron micrographs were taken with a FEI Teneo LoVac SEM in BSE mode. BSE images were taken for all the three samples at different locations from bottom to top of the build in XZ plane at different magnifications. EBSD analysis helped confirm the qualitative results of the SEM images using Oxford EBSD detector. $350 \times 350 \mu \mathrm{m}$ area maps with a $0.5 \mu \mathrm{m}$ step size and $8 \times 8$ binning were collected at the top, middle and bottom of each sample on the XZ plane.

TEM samples were prepared with a FEI Helios DualBeam ${ }^{\mathrm{TM}}$ (focused ion beam/SEM) from the bottom, middle, and top of the samples in the XZ plane. PED scans were carried out to obtain crystallographic information at nanoscale and quantify the dislocation density (density of dislocations) affected by the three different scan strategies using a MatLab code developed previously [13]. Scans for PED [14] with 2.5 x $4 \mu \mathrm{m}$ area were run on the TEM foils using ASTAR on FEI Tecnai G2-F20 STEM with $10 \mathrm{~nm}$ step size, 0.917 precession angle, 10 precessions per frame and 0.65 gamma. The camera length was set to $77 \mathrm{~mm}$ at smallest $\mathrm{C} 2$ aperture and smaller spot size.

\section{Results and Discussion}

Scanning electron microscopy was used to characterize microstructures from the builds made with three scan strategies along and across the build direction. A qualitative comparison of these samples from SEM showed that a columnar $\beta$ morphology was present in all of the builds. The raster scanning strategy (L5) has a finer morphology when compared to the two-point melting strategies (R5 and D5): the center of the builds have the following mean grain sizes: $6.36 \mu \mathrm{m}$ for L5, $9.81 \mu \mathrm{m}$ for D5 and $16.7 \mu \mathrm{m}$ for R5. All samples have both colony and basket-weave type microstructures; however, the fraction of colonies is different: $5 \%$ for L5, 30\% for D5 and $50 \%$ for R5 (all measured at the center of the builds in the XZ plane). An example of change in microstructures from process type and position is presented in Figure 4.

a

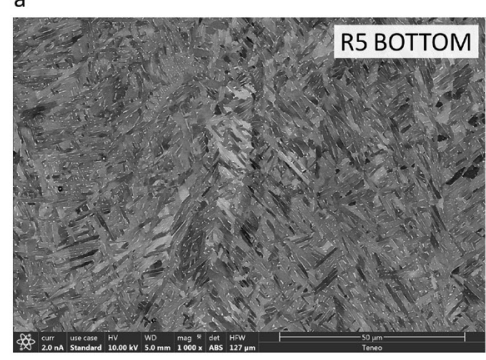

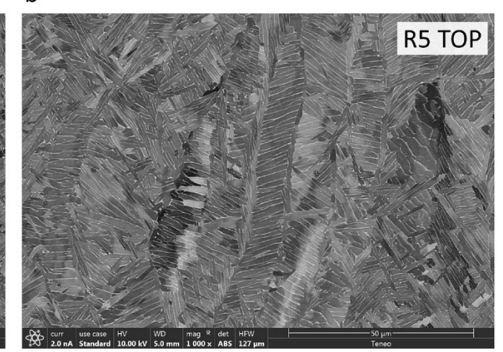

c

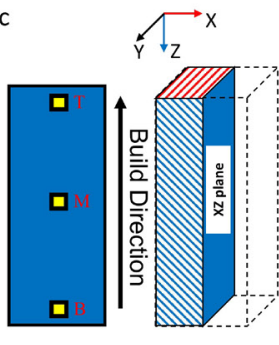

Figure 4: SEM images in BSE mode of AM Ti-6Al-4V samples showing colony and basket-weave type microstructures from (a) bottom of R5, (b) top of $\mathrm{R5}$ build and (c) schematic representation of sample orientation.

From SEM imaging and textures studies, it is evident that the bottom of all three samples is primarily basket-weave and it transitions to a more colony microstructure as we travel higher up the build. By the middle of the sample, the heating/cooling cycles appear to reach some steady state and as a result, the microstructure of the top and middle are largely the same.

Although comprehensive microstructural gradients were imaged across the different length scales using SEM, the crystallographic information is missing. As mentioned earlier, the crystallographic information is necessary to understand the effect of different scan strategies (or spatial variation of thermal gradients) on the solid-solid phase transformations. Therefore, we obtained micro-texture information is obtained using SEM-EBSD and nano-texture from PED ASTAR using TEM.

An analysis of texture for a grains from EBSD (Figure 5) shows changes along the build direction. Since the texture of $a$ is caused by two factors (i.e., the parent $\beta$ grain orientation and whether the microstructure is colony or basket-weave), a certain amount of local variation is to be expected. For example, when comparing the bottom and top of sample D5 shown in Figure 5(a), the difference in the IPF maps corresponds with the differences in the microstructural observations made in the SEM images. While this visual correspondence is useful, one advantage of conducting diffraction experiments is the representation of the crystallographic information as pole figures. In this texture analysis, two different types of diffraction experiments have been conducted - EBSD and neutron diffraction. The differences are that EBSD is a localized diffraction experiment, while neutron diffraction can be used to assess both bulk and local texture. While there are differences in the pole figures obtained from EBSD data in Figure 5(b) when compared with those from neutron diffraction data representing the bulk specimen in Figure $5(\mathrm{c})$, both sets of results are nominally consistent with a parent $\beta$ grain orientation whose (001) plane is nominally parallel with the $z$ direction and whose a precipitates obey a Burger's Orientation Relationship with the parent $\beta$ grains. For example, when looking at the (0002) for the neutron diffraction data, most of the information lies at $\sim 45^{\circ}$ away from a center position. We note that the center of this (0002) data is offset slightly from the center of the pole figure, corresponding with a "tilt" in the parent $\beta$ grains, consistent with observations previously made on optical images. The EBSD data, though representing the data from a different perspective (i.e., the y perspective) is also consistent, with the (0002) laying at 45 and $90^{\circ}$ to the center of the pole figure, and consistent with the aforementioned grain orientation and orientation relationship. 

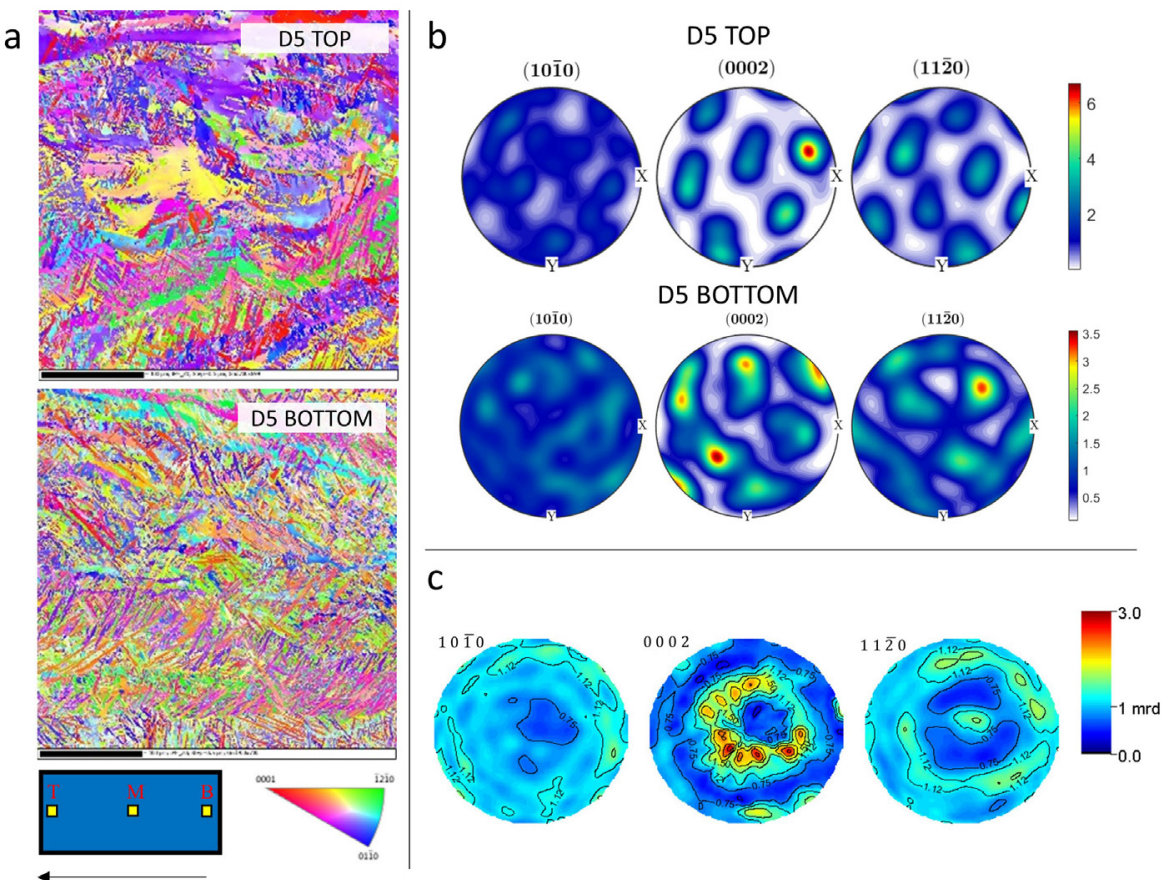

Figure 5: Results from Dehoff sample in XZ plane a) EBSD-SEM IPF maps for agrains of from top and bottom of the D5 build, b) Pole figures from EBSD scans from D5 and c) Pole figures from Neutron diffraction from D4 build (provided by Colorado School of Mines). Note: there is an orientation difference to data of Figure 4.

PED ASTAR experiments give crystallographic information at nanoscales but for the current work they are performed on thin TEM foils to obtain the quantitative information of the defect density (the density of dislocations in the printed samples). The PED scans are carried out for the TEM foils obtained from all the three samples from top, middle and bottom of the build are discussed below. The foils milled from XZ plane will lead to a foil containing the XY plane. Figure 6 shows preliminary data from one of the foils in PED ASTAR. The scans obtained are directly from the raw data without any cleaning steps with the optimized parameters mentioned in the previous section.

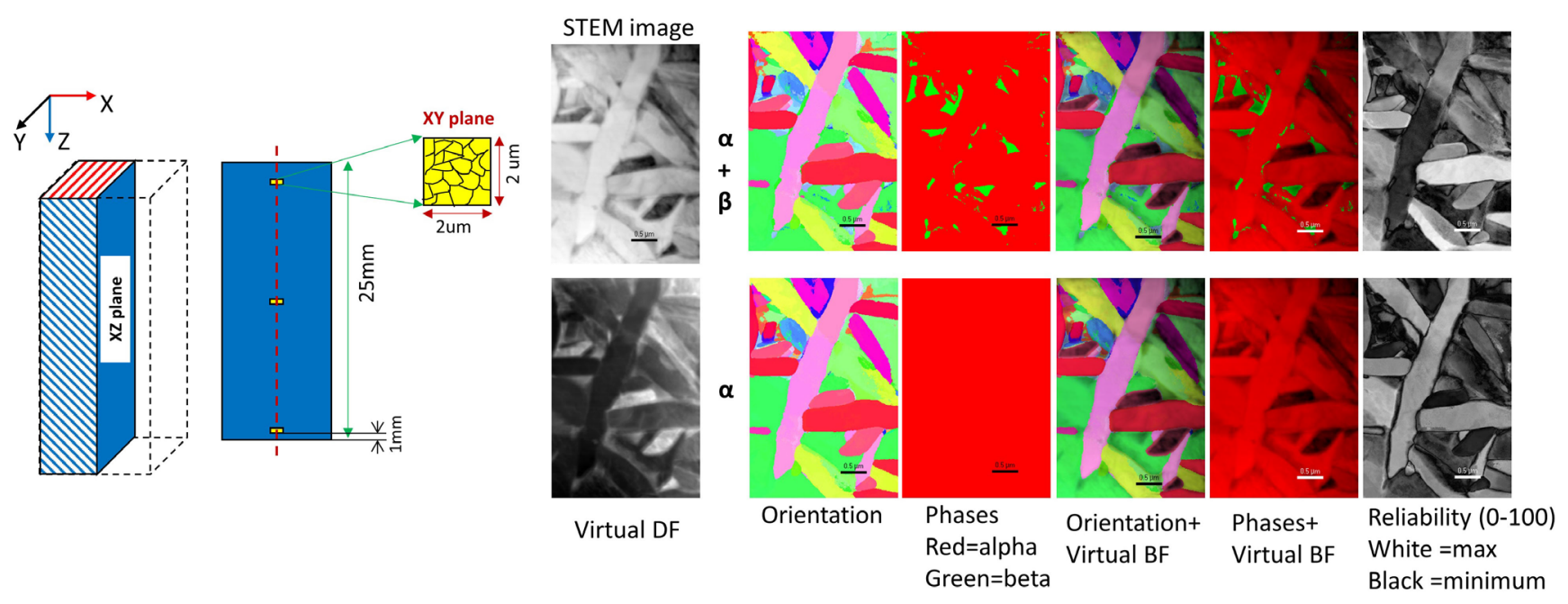

Figure 6: PED ASTAR scan from L5 sample bottom with optimized parameters. (a) Schematic to show the position of the TEM foils obtained from the AM build (b) Shows the texture information in two different rows. Top row presents $a+\beta$ texture whereas bottom row presents atexture

\section{Conclusion}

There is an interesting correlation between micrographs and texture information at different length scales and different build positions for all the three samples. The microstructure (both grain size and colony fraction) varies as a function of build height and scanning strategy, however a more detailed analysis is required, which is underway, to fully explain the results observed. 
The research is sponsored by the Department of the Navy, Office of Naval Research under ONR award number N00014-18-1-2794. Any opinions, findings, and conclusions or recommendations expressed in this material are those of the author(s) and do not necessarily reflect the views of the Office of Naval Research. Support was also given by Dr. Jonah Klemm-Toole of Colorado School of Mines in the data processing and analysis of neutron diffraction data.

We would like to acknowledge Dr. Suresh Babu (UTK and MDF, ONRL), Chase Joslin (MDF, ORNL), and James Ferguson (MDF, ORNL) who helped with the ARCAM machine in printing the builds.

The authors would also like to thank Dr. Sven Vogel at Los Alamos National Laboratory for his help in using the High-Pressure-Preferred Orientation neutron beam line to collect the bulk texture data presented here and in assisting with data processing.

\section{$\underline{\text { References }}$}

1. N. Hrabe, T. Quinn, Mater. Sci. Eng. A. 573 (2013) 264-270.

2. N. Hrabe, T. Quinn, Mater. Sci. Eng. A. 573 (2013) 271-277.

3. Y.-J. Liang, X.-J. Tian, Y.-Y. Zhu, J. Li, H.-M. Wang, Mater. Sci. Eng. A. 599 (2014) 242-246.

4. M.Y. Medoza, P. Samimi, D.A. Brice, B.W. Martin, M.R. Rolchigo, R. LeSar, P.C. Collins, Metall. Mater. Trans. A. 48A (2017) 35943605.

5. S. Tamirisakandala, R.B. Bhat, J.S. Tiley, D.B. Miracle, Scr. Mater. 53 (2005) 1421-1426.

6. M.J. Bermingham, S.D. McDonald, K. Nogita, D.H. St. John, M. S. Dargusch, Scr. Mater. 59 (2008) 538-541.

7. P.C. Collins, D.A. Brice, P. Samimi, I. Ghamarian, H.L. Fraser, Annu. Rev. Mater. Res. 46 (2016) 63-91.

8. R. Banerjee, S. Nag, S. Samuel, H.L. Fraser: J. Biomed, Mater. Res. A. 78 (2006) 298-305.

9. S. Nag, S. Samuel, A. Puthucode, R. Banerjee, Mater. Charact. 60 (2009) 106-113.

10. D. Rosenthal, Trans. of ASME. 68 (1946) 849-866.

11. A. Plotkowski, M.M. Kirka, S.S. Babu, Additive Manufacturing. 18 (2017) 256-268.

12. M Shafiqur Rahman, P.J. Schilling, P.D. Herrington, U.K. Chakravarty, J. Eng. Mater. Technol. 141(4) (2019) 041006-041006-12.

13. I. Ghamarian, P. Samimi, Y. Liu, B. Poorganji, V.K. Vasudevan, P.C. Collins, Mater. Charact. 113 (2016) 222-231.

14. D. Viladot, M. Veron, M. Gemmi, F. Peiro, J. Portillo, S. Estrade, J. Mendoza, N. Llorca-Isern, S. Nicopoulus. J Microsc 252 (1) (2013) 23-34 